\title{
Influences of dark energy and dark matter on gravitational time advancement
}

\author{
Samrat Ghosh ${ }^{\mathrm{a}}$, Arunava Bhadra ${ }^{\mathrm{b}}$ \\ High Energy and Cosmic Ray Research Center, University of North Bengal, Siliguri 734013, India
}

Received: 25 August 2015 / Accepted: 4 October 2015 / Published online: 16 October 2015

(C) The Author(s) 2015. This article is published with open access at Springerlink.com

\begin{abstract}
The effect of dark matter/energy on the gravitational time advancement (negative effective time delay) has been investigated considering a few dark energy/matter models including cosmological constant. It is found that dark energy gives only a (positive) gravitational time delay, irrespective of the position of the observer, whereas a pure Schwarzschild geometry leads to a gravitational time advancement when the observer is situated at a relatively stronger gravitational field point in the light trajectory. Consequently, there will be no time advancement effect at all at radial distances where the gravitational field due to dark energy is stronger than the gravitational field of the Schwarzschild geometry.
\end{abstract}

\section{Introduction}

The discovery of the acceleration of the universe's expansion [1-5] has led to the inclusion of a new component into the energy-momentum tensor of the universe having a negative pressure, the so-called dark energy component. On the other hand data from rotation curve surveys [6] and a few other observations $[7,8]$ require there to be a dominating component of matter in galaxies which is non-luminous or dark. Several other observations, which include the cosmic microwave background (CMB) measurements [9-12], baryon acoustic oscillations (BAO) [13-15], and lensing in clusters [16,17], support the existence of dark energy as well as the presence of a dark matter halo surrounding the Galactic disc. Consequently on large distance scales, astrophysical and cosmological phenomena are governed mainly by dark matter and dark energy.

The simplest candidate for dark energy is the cosmological constant $(\Lambda)$ : a constant energy density with equationof-state parameter $w=-1$ and the $\Lambda \mathrm{CDM}$ model where

\footnotetext{
a e-mail: samrat.ghosh003@gmail.com

be-mail: aru_bhadra@yahoo.com
}

CDM refers to cold dark matter, which is in accordance with all the existing cosmological observations $[18,19]$ such as the cosmic microwave background (CMB) anisotropies, the large scale structure, the scale of the baryonic acoustic oscillation in the matter power spectrum, and the luminosity distance of the supernovae type Ia; but it has a big theoretical problem-its size $\left(\sim 10^{-52} \mathrm{~m}^{-2}\right)$ is many orders of magnitudes below the expected vacuum energy density in the standard model of particle physics [20]. Hence many other theoretical explanations for the $\mathrm{DE}$ have been proposed in the literature in which the parameter $w$ evolves with time or is different from -1 such as the quintessence [21-23], k-essence [24-27], phantom field [28,29], and Chaplygin gas [30,31] models. There are also proposals for a modification of general relativity, which include scalar tensor theories [32] or $\mathrm{f}(\mathrm{R})$ gravity models [33], conformal gravity models [34,35], massive gravity theories [36] including Dvali-GabadadzePorrati (DGP) braneworld gravity $[37,38]$ models etc., which lead to late-time accelerated expansion without invoking any dark energy.

Like dark energy, there are also several candidates for dark matter [39] such as WIMPs, axions, sterile neutrinos etc. There are proposals for the modifications at the fundamental theoretical level as well, which include MOND [40-43], that suggest modifications in Newtonian dynamics. The evidence of the presence of non-baryonic dark matter from the CMB data, however, questions the MOND-like schemes. The conformal gravitational theory $[34,35]$, which is based on Weyl symmetry, also can explain flat rotation curves of galaxies without the need of dark matter.

Dark energy/matter is likely to affect the gravitational phenomena on all distance scales including the local scales. Several investigations have so far been made to estimate the influence of dark energy (mainly through cosmological constant) on different local gravitational phenomena, which include the three classical observables - the perihelion shift of planets $[44,45]$, gravitational bending of light [45-49], and grav- 
itational time delay (or Shapiro time delay) $[45,50,51]$. Due to the tiny value of $\Lambda$, the influence of dark energy has been to be found very small, not detectable by the ongoing experiments. Out of the local gravitational phenomena the effect of $\Lambda$ is found to be maximum in the case of perihelion precession of planets and the observations on perihelion precession of Mercury put an upper bound of $\Lambda \leq 10^{-42} \mathrm{~m}^{-2}$ [52]. On the other hand analysis of the perihelion precession of Mercury, Earth, and Mars also lead to a upper bound $3 \times 10^{-19}$ $\mathrm{g} / \mathrm{cm}^{3}$ for dark matter density $\left(\rho_{\mathrm{dm}}\right)$ [53], whereas the rotation curve data implies that $\rho_{\mathrm{dm}}$ in the Milky Way at the location of the solar system is $\rho_{\mathrm{dm}}=0.5 \times 10^{-24} \mathrm{~g} / \mathrm{cm}^{3}$ [54].

In this work we would like to examine the influence of dark energy and dark matter on gravitational time advancement. The gravitational time advancement effect takes place when the observer is situated at a stronger gravitational field with respect to the gravitational field encountered by the photon while traversing a certain path [55]. We found that dark energy and dark matter do affect the gravitational time advancement and though the magnitude of the effect is small, it induces an interesting observational consequence, at least in principle.

The organization of the paper is as follows. In the next section we discuss briefly the gravitational time advancement effect. The influence of dark energy and dark matter on gravitational time advancement are evaluated in Sect. 3. The results are discussed and finally we conclude in Sect. 4.

\section{Gravitational time advancement}

The gravitational time delay is one of the classical solar system tests of general relativity. The general perception as regards the gravitational time delay is that due to the influence of a gravitating object the average global speed of light is reduced from its special-relativistic value $c_{0}$ and hence the signal always suffers an additional time delay. But depending upon the position of the observer, the delay can as well be negative, which was called a gravitational time advancement [55]. To exemplify the effect let us consider light propagating in a gravitational field between two points A and B. Assuming the standard Schwarzschild geometry, i.e.

$\mathrm{d} s^{2}=-(1-2 \mu / r) \mathrm{d} t^{2}+(1-2 \mu / r) \mathrm{d} r^{2}+r^{2} / \mathrm{d} \Omega^{2}$,

the total coordinate time required for the round-trip journey between the points $\mathrm{A}$ and $\mathrm{B}$ (or between the points $\mathrm{B}$ to A and back) to the first order in $\mu=G M / c_{0}^{2}$ is given by [53]

$$
\begin{aligned}
c_{0} \Delta t_{\mathrm{AB}}= & 2\left(\sqrt{r_{\mathrm{A}}^{2}-r_{o}^{2}}+\sqrt{r_{\mathrm{B}}^{2}-r_{o}^{2}}\right)+ \\
& +4 \mu\left(\ln \frac{r_{\mathrm{A}}+\sqrt{r_{\mathrm{A}}^{2}-r_{o}^{2}}}{r_{o}}+\ln \frac{r_{\mathrm{B}}+\sqrt{r_{\mathrm{B}}^{2}-r_{o}^{2}}}{r_{o}}\right) \\
& +2 \mu\left[\left(\frac{r_{\mathrm{A}}-r_{o}}{r_{\mathrm{A}}+r_{o}}\right)^{1 / 2}+\left(\frac{r_{\mathrm{B}}-r_{o}}{r_{\mathrm{B}}+r_{o}}\right)^{1 / 2}\right]
\end{aligned}
$$

where $r_{\mathrm{A}}$ and $r_{\mathrm{B}}$ are the radial coordinates of the point $\mathrm{A}$ and $\mathrm{B}$, respectively, and $r_{o}$ is the closest distance to the gravitating object in the photon path.

Suppose the point $\mathrm{A}$ is located at a relatively much weaker gravitational field due to a mass $\mathrm{M}$ than the point $\mathrm{B}$ i.e. $r_{\mathrm{A}}>>$ $r_{\mathrm{B}}$ where $r_{\mathrm{A}}$ and $r_{\mathrm{B}}$ are the values of coordinate $r$ evaluated at the position $\mathrm{A}$ and $\mathrm{B}$, respectively. Hence the proper time for transmission and the reception of the signal to be measured by the observer at the point $\mathrm{A}$ is

$$
\begin{aligned}
c_{0} \Delta \tau_{\mathrm{AB}} \simeq & \left(1-\frac{\mu}{r_{\mathrm{A}}}\right) \Delta t_{\mathrm{AB}} \simeq 2\left(\sqrt{r_{\mathrm{A}}^{2}-r_{o}^{2}}+\sqrt{r_{\mathrm{B}}^{2}-r_{o}^{2}}\right) \\
& +4 \mu\left(\ln \frac{r_{\mathrm{A}}+\sqrt{r_{\mathrm{A}}^{2}-r_{o}^{2}}}{r_{o}}+\ln \frac{r_{\mathrm{B}}+\sqrt{r_{\mathrm{B}}^{2}-r_{o}^{2}}}{r_{o}}\right) \\
& +2 \mu\left[\left(\frac{r_{\mathrm{A}}-r_{o}}{r_{\mathrm{A}}+r_{o}}\right)^{1 / 2}+\left(\frac{r_{\mathrm{B}}-r_{o}}{r_{\mathrm{B}}+r_{o}}\right)^{1 / 2}\right]
\end{aligned}
$$

In the above expression the first term on the right hand side is the usual special-relativistic time of travel. The remaining two terms are general-relativistic corrections. As a result the observed time will be higher than the time taken between transmission and the reception in the absence of a gravitating object, which is the well-known gravitational time delay.

Now let us consider the case that the observer is at the point $\mathrm{B}$ instead of the point $\mathrm{A}$. In that case the proper time between transmission and the reception of the signal to be measured by the observer will be [55]

$$
\begin{aligned}
c_{0} \Delta \tau_{\mathrm{AB}} \simeq & \left(1-\frac{\mu}{r_{\mathrm{B}}}\right) \Delta t_{\mathrm{AB}} \simeq 2\left(\sqrt{r_{\mathrm{A}}^{2}-r_{o}^{2}}+\sqrt{r_{\mathrm{B}}^{2}-r_{o}^{2}}\right) \\
& +4 \mu\left(\ln \frac{r_{\mathrm{A}}+\sqrt{\left(r_{\mathrm{A}}^{2}-r_{o}^{2}\right)}}{r_{o}}+\ln \frac{r_{\mathrm{B}}+\sqrt{\left(r_{\mathrm{B}}^{2}-r_{o}^{2}\right)}}{r_{o}}\right) \\
& +2 \mu\left[\left(\frac{r_{\mathrm{A}}-r_{o}}{r_{\mathrm{A}}+r_{o}}\right)^{1 / 2}+\left(\frac{r_{\mathrm{B}}-r_{o}}{r_{\mathrm{B}}+r_{o}}\right)^{1 / 2}\right] \\
& -2 \mu\left(\frac{\left(\sqrt{r_{\mathrm{A}}^{2}-r_{o}^{2}}+\sqrt{r_{\mathrm{B}}^{2}-r_{o}^{2}}\right)}{r_{\mathrm{B}}}\right) .
\end{aligned}
$$

Due to the last term of the right hand side of the above expression, which is the dominating one among the generalrelativistic correction terms, the time taken between trans- 
mission and the reception will be reduced from the usual special-relativistic time of travel when the distance between $A$ and $B$ exceeds a certain value. This effect is known as the gravitational time advancement (negative time delay), which arises because of the clock running differently at different positions in the gravitational field.

\section{Influence of dark energy/matter on gravitational time advancement}

In the presence of dark energy the exterior space-time of a spherically symmetric mass distribution is no longer described by the Schwarzschild geometry, but by some modification of the Schwarzschild metric. For instance if dark energy is the cosmological constant, the exterior static spacetime will be the Schwarzschild-de Sitter (SDS) space-time.

Here we shall consider a general static spherically symmetric metric of the form

$\mathrm{d} s^{2}=-\mathrm{B}(r) \mathrm{d} t^{2}+A(r) \mathrm{d} r^{2}+r^{2} / \mathrm{d} \Omega^{2}$

with

$\mathrm{B}(r)=1-2 m / r-a \Lambda r^{n} / 3$

and

$A(r)=\left(1-2 m / r-\Lambda r^{n} / 3\right)^{-1}$

where $a$ and $\Lambda$ are constants. Different choices of $\mathrm{n}$ and a lead to different models of dark energy.

Case 1: With $n=1 / 2, a=2$, and $\Lambda= \pm \sqrt{G M / r_{c}^{2}}$, the model represents the gravitational field of a spherically symmetric matter distribution on the background of an accelerating universe in the Dvali-Gabadadze-Porrati (DGP) braneworld gravity, provided only leading terms are considered [56]. $r_{c}$ is the crossover scale beyond which gravity becomes five dimensional.

Case 2: For the choice $n=1, a=1$, and negative $\Lambda$, the model well describes the gravitational potential due to a central matter distribution plus dark matter $[34,35,57]$.

Case 3: If $n=3 / 2, a=2 / 3$, and $\Lambda=-m_{g}^{2} \sqrt{\frac{2 G M}{13 c^{2}}}$, the model corresponds to the non-perturbative solution of a massive gravity theory (an alternative description of accelerating expansion of the universe) [58] where $m_{g}$ is the mass of graviton.

Case 4: When $a=1, n=2$, and $m=\mu$ the above metric describes the Schwarzschild-de Sitter (SDS) or Kotler space-time, which is the exterior space-time due to a static spherically symmetric mass distribution in the presence of the cosmological constant $\Lambda$ [59].

\subsection{General trajectory}

Now let us suppose that a light beam is moving between two points A and B in the gravitational field of Eqs. (5-7). The expression for the coordinate time required for light rays to traverse the distance $r_{o}$ to $r$, where $r_{o}$ is the closest distance from the gravitating object over the trajectory can be obtained from the geodesic equations, given by

$\delta t=\int_{r_{o}}^{r} \sqrt{P\left(r, r_{o}\right)} \mathrm{d} r$

where

$P\left(r, r_{o}\right)=\frac{\mathrm{A}(r) / \mathrm{B}(r)}{1-\frac{r_{o}^{2}}{r^{2}} \frac{\mathrm{B}(r)}{\mathrm{B}\left(r_{o}\right)}}$.

For a general power index ( $n$ ) of $\Lambda$ in Eq. (5), the above equation after integration can only be expressed in terms of hyper-geometric functions and thereby are not very useful. However, for $n=1$ and $n=2$, the integral can be written in a handy form, particularly when higher order terms in $\mathrm{M}$ and $\Lambda$ are ignored. The extra coordinate time delay $\left(\delta t_{1}^{\Lambda}\right)$ induced by the dark sector terms in Eq. (8) is given, for $n=1$ and $\Lambda=-\Lambda$, by

$$
\begin{aligned}
\delta t_{1}^{\Lambda}= & -(a+1) \frac{\Lambda}{12}\left(r \sqrt{r^{2}-r_{o}^{2}}+r_{o}^{2} \ln \left(r+\sqrt{r^{2}-r_{0}^{2}}\right)\right) \\
& -\frac{a \Lambda r_{o}^{2}}{6}\left(\ln \left(r+\sqrt{r^{2}-r_{0}^{2}}\right)-\sqrt{\frac{r-r_{o}}{r+r_{o}}}\right)
\end{aligned}
$$

while for $n=2$ we have

$\delta t_{2}^{\Lambda}=(a+1) \frac{\Lambda}{18}\left(\left(r^{2}+2 r_{o}^{2}\right) \sqrt{r^{2}-r_{o}^{2}}\right)-\frac{a \Lambda r_{o}^{2}}{6} \sqrt{r^{2}-r_{o}^{2}}$,

and for general $n(n \neq 1)$ when $r_{\mathrm{A}}>>r_{o}$ and $r_{\mathrm{B}}>>r_{o}$,

$\delta t_{n}^{\Lambda} \simeq \frac{(a+1) \Lambda}{6(n+1)} r^{n+1}-\frac{(a-1) \Lambda}{12(n-1)} r^{n-1} r_{o}^{2}+O\left(r_{o}^{4}\right)$.

Hence the proper time between the transmission and the reception of the signal to be measured by the observer at point B will be for $n=1$

$$
\begin{array}{rl}
c_{0} \Delta \tau_{1} \simeq 2 & 2\left(\sqrt{r_{\mathrm{A}}^{2}-r_{o}^{2}}+\sqrt{r_{\mathrm{B}}^{2}-r_{o}^{2}}\right) \\
& +4 \mu\left(\ln \frac{r_{\mathrm{A}}+\sqrt{r_{\mathrm{A}}^{2}-r_{o}^{2}}}{r_{o}}+\ln \frac{r_{\mathrm{B}}+\sqrt{r_{\mathrm{B}}^{2}-r_{o}^{2}}}{r_{o}}\right)
\end{array}
$$




$$
\begin{aligned}
& +2 \mu\left[\left(\frac{r_{\mathrm{A}}-r_{o}}{r_{\mathrm{A}}+r_{o}}\right)^{1 / 2}+\left(\frac{r_{\mathrm{B}}-r_{o}}{r_{\mathrm{B}}+r_{o}}\right)^{1 / 2}\right] \\
& -(a+1) \frac{\Lambda}{12}\left(r_{\mathrm{A}} \sqrt{r_{\mathrm{A}}^{2}-r_{o}^{2}}+r_{o}^{2} \ln \left(r_{\mathrm{A}}+\sqrt{r_{\mathrm{A}}^{2}-r_{o}^{2}}\right)\right) \\
& +\frac{a \Lambda r_{o}^{2}}{6}\left(\sqrt{\frac{r_{\mathrm{A}}-r_{o}}{r_{\mathrm{A}}+r_{o}}}-\ln \left(r_{\mathrm{A}}+\sqrt{r_{\mathrm{A}}^{2}-r_{o}^{2}}\right)\right) \\
& -(a+1) \frac{\Lambda}{12}\left(r_{\mathrm{B}} \sqrt{r_{\mathrm{B}}^{2}-r_{o}^{2}}+r_{o} \ln \left(r_{\mathrm{B}}+\sqrt{r_{\mathrm{B}}^{2}-r_{o}^{2}}\right)\right) \\
& +\frac{a \Lambda r_{o}^{2}}{6}\left(\sqrt{\left.\frac{r_{\mathrm{B}}-r_{o}}{r_{\mathrm{B}}+r_{o}}-\ln \left(r_{\mathrm{B}}+\sqrt{r_{\mathrm{B}}^{2}-r_{o}^{2}}\right)\right)}\right. \\
& -2\left(\frac{\mu}{r_{\mathrm{B}}}-\frac{a \Lambda r_{\mathrm{B}}}{3}\right)\left(\sqrt{r_{\mathrm{A}}^{2}-r_{o}^{2}}+\sqrt{r_{\mathrm{B}}^{2}-r_{o}^{2}}\right)
\end{aligned}
$$

Usually for observing a time advancement effect, $r_{o}=r_{\mathrm{B}}$. Further for describing a flat rotation curve, $a$ has been chosen as 1 . Hence the above equation reduces to

$$
\begin{aligned}
c_{0} \Delta \tau_{1} \simeq & 2 \sqrt{r_{\mathrm{A}}^{2}-r_{\mathrm{B}}^{2}}+4 \mu \ln \left(\frac{r_{\mathrm{A}}+\sqrt{r_{\mathrm{A}}^{2}-r_{\mathrm{B}}^{2}}}{r_{\mathrm{B}}}\right) \\
& +2 \mu\left(\frac{r_{\mathrm{A}}-r_{\mathrm{B}}}{r_{\mathrm{A}}+r_{\mathrm{B}}}\right)^{1 / 2} \\
& -\frac{\Lambda}{6}\left(r_{\mathrm{A}} \sqrt{r_{\mathrm{A}}^{2}-r_{\mathrm{B}}^{2}}+r_{\mathrm{B}}^{2} \ln \left(r_{\mathrm{A}}+\sqrt{r_{\mathrm{A}}^{2}-r_{\mathrm{B}}^{2}}\right)\right) \\
& -\frac{\Lambda}{6} r_{\mathrm{B}}^{2}\left(\sqrt{\frac{r_{\mathrm{A}}-r_{\mathrm{B}}}{r_{\mathrm{A}}+r_{\mathrm{B}}}}-\ln \left(r_{\mathrm{A}}+\sqrt{r_{\mathrm{A}}^{2}-r_{\mathrm{B}}^{2}}\right)\right) \\
& -2\left(\frac{\mu}{r_{\mathrm{B}}}-\frac{\Lambda r_{\mathrm{B}}}{3}\right) \sqrt{r_{\mathrm{A}}^{2}-r_{\mathrm{B}}^{2}} .
\end{aligned}
$$

When $r_{\mathrm{A}}>r_{\mathrm{B}}$, the above equation transforms to

$$
\begin{aligned}
c_{0} \Delta \tau_{1} \simeq & 2 r_{\mathrm{A}}-2 \mu\left(\frac{r_{\mathrm{A}}}{r_{\mathrm{B}}}-2 \ln \left(\frac{2 r_{\mathrm{A}}}{r_{\mathrm{B}}}\right)-1\right) \\
& -\frac{\Lambda}{6}\left(r_{\mathrm{A}}^{2}+2 r_{\mathrm{B}}^{2} \ln 2 r_{\mathrm{A}}-4 r_{\mathrm{A}} r_{\mathrm{B}}\right) .
\end{aligned}
$$

Similarly for $n=2$ with $r_{o}=r_{\mathrm{B}}$

$$
\begin{aligned}
c_{0} \Delta \tau_{2} \simeq & 2 \sqrt{r_{\mathrm{A}}^{2}-r_{\mathrm{B}}^{2}} \\
& +4 \mu \ln \left(\frac{r_{\mathrm{A}}+\sqrt{r_{\mathrm{A}}^{2}-r_{\mathrm{B}}^{2}}}{r_{\mathrm{B}}}\right)+2 \mu\left(\frac{r_{\mathrm{A}}-r_{\mathrm{B}}}{r_{\mathrm{A}}+r_{\mathrm{B}}}\right)^{1 / 2} \\
& +(a+1) \frac{\Lambda}{18}\left(r_{\mathrm{A}}^{2}+2 r_{\mathrm{B}}^{2}\right) \sqrt{r_{\mathrm{A}}^{2}-r_{\mathrm{B}}^{2}} \\
& -\frac{a \Lambda r_{\mathrm{B}}^{2}}{6} \sqrt{r_{\mathrm{A}}^{2}-r_{\mathrm{B}}^{2}}-2\left(\frac{\mu}{r_{\mathrm{B}}}+\frac{a \Lambda r_{\mathrm{B}}^{2}}{3}\right) \sqrt{r_{\mathrm{A}}^{2}-r_{\mathrm{B}}^{2}},
\end{aligned}
$$

which for $r_{\mathrm{A}}>>r_{\mathrm{B}}$ becomes

$$
\begin{aligned}
c_{0} \Delta \tau_{2} \simeq & 2 r_{\mathrm{A}}+2 \mu\left(2 \ln \left(\frac{2 r_{\mathrm{A}}}{r_{\mathrm{B}}}\right)+1-\frac{r_{\mathrm{A}}}{r_{\mathrm{B}}}\right) \\
& \left.+\frac{\Lambda}{18}\left((a+1) r_{\mathrm{A}}^{3}+a r_{\mathrm{A}} r_{\mathrm{B}}^{2}(2-13 a)\right)-2 \frac{a r_{\mathrm{A}} r_{\mathrm{B}}}{3}\right),
\end{aligned}
$$

and for general $n$

$$
\begin{aligned}
c_{0} \Delta \tau_{n} \simeq & 2 r_{\mathrm{A}}+2 \mu\left(2 \ln \left(\frac{2 r_{\mathrm{A}}}{r_{\mathrm{B}}}\right)+1-\frac{r_{\mathrm{A}}}{r_{\mathrm{B}}}\right) \\
& +\frac{(a+1) \Lambda}{6(n+1)} r^{n+1}-\frac{(a-1) \Lambda}{12(n-1)} r^{n-1} r_{o}^{2} \\
& -\frac{2 a \Lambda r_{\mathrm{A}} r_{\mathrm{B}}^{n}}{3} .
\end{aligned}
$$

Unless the $\Lambda$ effect dominates over the pure Schwarzschild effect, the net time delay will be negative in all the above cases resulting in time advancement.

\subsection{Small distance travel}

Let us suppose a light beam is moving from a point on the Earth surface (B) $(R, \theta, \phi)$, where the radius of Earth is denoted as $R_{E}$, to a nearby point with coordinates $\mathrm{C}(R+$ $\Delta R, \theta, \phi)$ and reflects back to the transmitter position (B). The light signal will travel a null curve of space-time, satisfying $\mathrm{d} s^{2}=0$. Then the proper distance between point $\mathrm{B}$ and point $\mathrm{C}$ is given by

$$
\begin{aligned}
\Delta L_{\mathrm{BC}}= & \int_{R}^{R+\Delta R}\left(1-2 m / r-\Lambda r^{n} / 3\right)^{-1 / 2} \mathrm{~d} r \\
\simeq & \Delta R\left[1+\frac{m}{R}-\frac{m \Delta R}{2 R^{2}}\right. \\
& +\frac{\Lambda R^{n}}{6}\left(1+\frac{n \Delta R}{2 R}+\frac{n(n-1) \Delta R^{2}}{6 R^{2}}\right) \\
& \left.+\frac{3 m^{2}}{2 R^{2}}+\frac{m \Lambda R^{n-1}}{2}\left(1+\frac{(n-1) \Delta R}{2 R}\right)\right] .
\end{aligned}
$$

The coordinate time interval in transmitting a light signal from $\mathrm{B}$ to $\mathrm{C}$ and back, is given by

$$
\begin{aligned}
\Delta t= & 2 \int_{R}^{R+\Delta R}\left(1-\frac{2 m}{r}-\frac{\Lambda r^{n}}{3}\right)^{-1 / 2}\left(1-\frac{2 m}{r}-\frac{a \Lambda r^{n}}{3}\right)^{-1 / 2} \mathrm{~d} r \\
\simeq & 2 L_{\mathrm{BC}}\left[1+\frac{m}{R}+\frac{3 m^{2}}{2 R^{2}}+\frac{\Lambda a R^{n}}{6}\left(1+\frac{n \Delta R}{2 R}+\frac{n(n-1) \Delta R^{2}}{6 R^{2}}\right)\right. \\
& +m \Lambda\left(R^{n-1}\left(1+\frac{(n-1) \Delta R}{2 R}\right)\left(\frac{2 a}{3}+\frac{1}{6}\right)\right. \\
& \left.\left.-\frac{(a+1) R^{n-1}}{6}\left(1+\frac{n \Delta R}{2 R}\right)+\frac{R^{n-2}(a+1) \Delta R}{12}\right)-\frac{m \Delta R}{2 R^{2}}\right] .
\end{aligned}
$$


The observer at $\mathrm{B}$ will experience that coordinate time interval in proper time to be measured by the observer at $B$ between transmission and reception of the signal as given by

$$
\begin{aligned}
\Delta \tau_{1}= & \left(1-\frac{2 m}{R}-\frac{a \Lambda R^{n}}{3}\right)^{1 / 2} \Delta t \\
\simeq & 2 L_{\mathrm{BC}}\left[1+\frac{\Lambda a R^{n}}{6}\left(\frac{n \Delta R}{2 R}+\frac{n(n-1) \Delta R^{2}}{6 R^{2}}\right)\right. \\
& +m \Lambda\left(R^{n-1}\left(1+\frac{(n-1) \Delta R}{2 r}\right)\left(\frac{2 a}{3}+\frac{1}{6}\right)\right. \\
& -\frac{R^{n-1}(a+1)}{6}\left(1+\frac{n \Delta R}{2 R}\right)+\frac{R^{n-2}(2 a+1) \Delta R}{12} \\
& \left.\left.-\frac{a R^{n-1}}{6}\left(3+\frac{n \Delta R}{2 R}\right)\right)-\frac{m \Delta R}{2 R^{2}}\right]
\end{aligned}
$$

In deriving the above equations, the higher order terms in $\Lambda$ and $m^{2} \Lambda, m^{3}, m^{2} \frac{\Delta R^{2}}{R^{2}}$, and terms of higher order in $m$ have been neglected.

\section{Discussion and conclusion}

Dark energy has a significantly different kind of influence on gravitational time advancement than that of pure Schwarzschild geometry. The time advancement effect is entirely due to the pure Schwarzschild geometry, while dark energy leads only to a time delay effect, which means the gravitational time advancement effect will be reduced in the presence of dark energy. When $\Lambda r_{\mathrm{A}}^{2}>2 \mu / r_{\mathrm{B}}$, there no time advancement at all. So in principle the time advancement effect should be able to identify dark matter clearly.

In contrast the conformal theory description of a flat rotation curve suggests a large time advancement effect. The fitting of galactic rotation curves suggests $\Lambda / 3=-(5.42 \times$ $\left.10^{-42} \frac{M}{M_{\odot}}+3.06 \times 10^{-30}\right) \mathrm{cm}^{-1}$ [60]. Therefore, in our galaxy, the dark matter potential should start dominating over the luminous matter contribution (pure Schwarzschild part) at distances larger than about $30 \mathrm{kpc}$. Hence at distances beyond the $\sim 30 \mathrm{kpc}$ time advancement effect will be quite large. The experimental realization to examine the gravitational time advancement effect at such distances is a challenging issue.

Here it is worthwhile to mention that the gravitational time advancement effect has not been experimentally verified yet, but it should not be very difficult to test the effect. This is because the magnitude of the time advancement effect is reasonably large. In fact, gravitational time advancement is a much stronger effect than gravitational time delay when large distances are involved. However, time delay has the advantage of probing stronger gravity. In the solar system tests of gravitation, time delay measurements mainly rely on the passage of radiation grazing the sun, and thereby the solar gravitational potential at the surface of the sun comes into play. In such a situation the time delay is about $240 \mu \mathrm{s}$, whereas the total special-relativistic travel time between the earth and the sun is about $1000 \mathrm{~s}$, which means the gravitational time delay is about a $2 \times 10^{-7}$ part of the total travel time. For testing gravitational time advancement from the earth or its surroundings, on the other hand, the solar gravitational potential at the position of earth will be applicable and when light propagates from the earth to say Pluto and back, the time advancement will be about $1 \mathrm{~ms}$ over a total propagation time of $50,000 \mathrm{~s}$ i.e. here the time advancement is about a $0.2 \times 10^{-7}$ part of the total travel time, which is just one order smaller than the time delay caused by the sun and hence is detectable. Note that the above estimates need to be corrected taking into account the variations in round-trip travel time due to the orbital motion of the target relative to the Earth by using radar-ranging or any other similar kind of data. Since gravity cannot be switched off, one does not have access to a special-relativistic propagation of a photon against which the time delay is to be measured. Therefore, the variation of the time delay is measured as a function of distance to verify the radial profile of Eq. (3). A similar check can be made for the time advancement also.

The future missions, such as the Beyond Einstein Advanced Coherent Optical Network (BEACON) [61] or the GRACE Follow-On (GRACE-FO) mission [62], will probe the gravitational field of the Earth with unprecedented accuracy. The BEACON mission will employ four small spacecraft equipped with laser transceivers and the spacecraft will be placed in a circular Earth orbit at a radius of $80,000 \mathrm{~km}$. All the six distances between the spacecraft will be measured to high accuracy $(\sim 0.1 \mathrm{~nm})$, out of which one diagonal laser trajectory will be very close to the Earth and thereby pick up the gravitational time delay effect. If the distance between the spacecraft and the Earth is also measured by an Earth bound observer and compared with distances measured by the spacecraft, the time advancement effect may be revealed from the measurements. The GRACE-FO, which is scheduled for launch in 2017 , will be equipped with a laser ranging interferometer and is expected to provide a range with an accuracy of $1 \mathrm{~nm}$. With such a level of accuracy general-relativistic effects may become significant [63]. It is, therefore, important to examine whether the time advancement can have any significant effect on the observables of GRACE-FO.

To probe dark matter through its influence on the gravitational time advancement properly, one is required to observe a time advancement (delay) effect at distance $\sim 30 \mathrm{kpc}$ or beyond. For probing dark energy, observations are to be made at even higher distances. This is currently not feasible. At present, observations can be made only from the Earth 
or from its neighborhood via a satellite/space station. So strategies to be developed for observing the time advancement/delay effect at other distances may be some indirect means. This would be a very challenging task.

For small distance travel, the time advancement effect is a second order effect, unlike the long distance travel where the time advancement occurs due to first order effect. However, since the time advancement effect is cumulative in nature, if a light beam is allowed to travel, say, from the Earth surface radially upwards to a nearby point large number of times it (the light beam), this should acquire a time advancement of reasonable magnitude when observed from the Earth surface and should be measurable.

In summary, we investigate the influence of dark matter/energy on gravitational time advancement. We obtain analytical expressions for the time advancement to first order in $M$ and $\Lambda$ where $\Lambda$ is the parameter describing the strength of the dark matter/energy. From our results it is found that dark energy leads to a gravitational time delay only, whereas a pure Schwarzschild metric gives both a time delay and a time advancement (negative effective time delay) depending on the position of the observer.

The present finding suggests that in principle the measurements of gravitational time advancement at large distances can verify the dark matter and a few dark energy models or put an upper limit on the dark matter/energy parameter.

Acknowledgments The authors are thankful to an anonymous reviewer for encouraging comments and drawing attention to few useful references.

Open Access This article is distributed under the terms of the Creative Commons Attribution 4.0 International License (http://creativecomm ons.org/licenses/by/4.0/), which permits unrestricted use, distribution, and reproduction in any medium, provided you give appropriate credit to the original author(s) and the source, provide a link to the Creative Commons license, and indicate if changes were made. Funded by SCOAP ${ }^{3}$.

\section{References}

1. A. Riess et al., Astron. J. 116, 1009 (1998)

2. S. Perlmutter et al., ApJ 517, 565 (1999)

3. M. Hicken, W.M. Wood-Vasey, S. Blondin et al., ApJ 700, 1097 (2009)

4. N. Suzuki, D. Rubin, C. Lidman et al., ApJ 746, 85 (2012)

5. M. Kowalski, D. Rubin, G. Aldering et al., ApJ 686, 749 (2008)

6. W.J.G. de Blok, F. Walter, E. Brinks, C. Trachternach, S.-H. Oh, R.C. Kenni-cutt, Astron. J. 136, 2648 (2008)

7. V. Trimble, Annu. Rev. Astron. Astrophys. 25, 425 (1987)

8. G. D'Amico, M. Kamionkowski, K. Sigurdson, arXiv:0907.1912v1 (2009)

9. P. de Bernardis, P.A.R. Ade, J.J. Bock et al., Nature 404, 955 (2000)

10. E. Komatsu, J. Dunkley, M.R. Nolta et al., ApJS 180, 330 (2009)

11. P.A.R. Ade, N. Aghanim, C. Armitage-Caplan et al. (Planck Collaboration), Astron. Astrophys. 571, A16 (2014)

12. P.A.R. Ade, N. Aghanim, C. Armitage-Caplan et al. (Planck Collaboration), Astron. Astrophys. 571, A23 (2014)
13. D.J. Eisenstein, I. Zehavi, D.W. Hogg et al., ApJ 633, 560 (2005)

14. L. Anderson, E. Aubourg, S. Bailey et al., MNRAS 427, 3435 (2012)

15. A. Slosar et al., J. Cosmol. Astropart. Phys. 4, 26 (2013)

16. S.W. Allen, D.A. Rapetti, R.W. Schmidt et al., MNRAS 383, 879 (2008)

17. T. Schrabback, J. Hartlap, B. Joachimi et al., Astron. Astrophys. 516, A63 (2010)

18. S.M. Carroll, Living Rev. Relativ. 4, 1 (2001)

19. P. Peebles, B. Ratra, Rev. Mod. Phys. 75, 559 (2003)

20. S. Weinberg, Rev. Mod. Phys. 61, 1 (1989)

21. B. Ratra, P. Peebles, Phys. Rev. D 37, 3406 (1988)

22. R. Caldwell, R. Dave, P.J. Steinhardt, Phys. Rev. Lett. 80, 1582 (1998)

23. I. Zlatev, L.-M. Wang, P.J. Steinhardt, Phys. Rev. Lett. 82, 896 (1999)

24. C. Armendariz-Picon, T. Damour, V.F. Mukhanov, Phys. Lett. B 458, 209 (1999)

25. C. Armendariz-Picon, V.F. Mukhanov, P.J. Steinhardt, Phys. Rev. Lett. 85, 4438 (2000)

26. C. Armendariz-Picon, V.F. Mukhanov, P.J. Steinhardt, Phys. Rev. D 63, 103510 (2001)

27. J. Garriga, V.F. Mukhanov, Phys. Lett. B 458, 219 (1999)

28. R.R. Caldwell, Phys. Lett. B 545, 23 (2002)

29. F. Hoyle, Mon. Not. R. Astron. Soc. 108, 372 (1948)

30. A.Y. Kamenshchik, U. Moschella, V. Pasquier, Phys. Lett. B 511, $265(2001)$

31. M. Bento, O. Bertolami, A. Sen, Phys. Rev. D 66, 043507 (2002)

32. T. Damour, G. Esposito-Farèse, Class. Quantum Gravity 9, 2093 (1992)

33. A. De Felice, S. Tsujikawa, Living Rev. Relativ. 13, 3 (2010)

34. P.D. Mannheim, D. Kazanas, Astrophys. J. 342, 635 (1989)

35. D. Kazanas, P.D. Mannheim, Astrophys. J. Suppl. 76, 431 (1991)

36. C. de Rham, Living Rev. Relativ. 17, 7 (2014)

37. G. Dvali, G. Gabadadze, M. Porrati, Phys. Lett. B 485, 208 (2000)

38. A. Lue, Phys. Rep. 423, 1 (2006)

39. J.L. Feng, Annu. Rev. Astron. Astrophys. 48, 495 (2010)

40. M. Milgrom, Astrophys. J. 270, 365 (1983)

41. J.D. Bekenstein, M. Milgrom, Astrophys. J. 286, 7 (1984)

42. J.D. Bekenstein, Contemp. Phys. 47, 387 (2006)

43. P.G. Ferreira, G. Starkmann, Science 326, 812 (2009)

44. J.N. Islam, Phys. Lett. A 97, 239 (1983)

45. V. Kagramanova, J. Kunz, C. Lämmerzahl, Phys. Lett. B 634, 465 (2006)

46. A. Bhadra, S. Biswas, K. Sarkar, Phys. Rev. D 82, 063003 (2010)

47. W. Rindler, M. Ishak, Phys. Rev. D 76, 043006 (2007)

48. M. Sereno, Phys. Rev. D 77, 043004 (2008)

49. M. Sereno, Phys. Rev. Lett. 102, 021301 (2009)

50. H. Asada, Phys. Lett. B 661, 78 (2008)

51. B. Chen, R. Kantowski, X. Dai, Phys. Rev. D 82, 043005 (2010)

52. S. Liang, Y. Xie, Res. Astron. Astrophys. 14, 527 (2014)

53. I.B. Khriplovich, E.V. Pitjeva, Int. J. Mod. Phys. D 15, 615 (2006)

54. G. Bertone, D. Merritt, Mod. Phys. Lett. A 20, 1021 (2005)

55. A. Bhadra, K.K. Nandi, Gen. Relativ. Gravity 42, 293 (2010)

56. A. Lue, G. Starkman, Phys. Rev. D 67, 064002 (2003)

57. D. Grumiller, Phys. Rev. Lett. 105, 211303 (2010)

58. A.I. Vainshtein, Phys. Lett. B 39, 393 (1972)

59. F. Kottler, Ann. Phys. (Leipzig) 361, 401 (1918)

60. P.D. Mannheim, J.G. O’Brien, Phys. Rev. Lett. 106, 121101 (2011)

61. S.G. Turyshev, B. Lane, M. Shao, A. Girerd, Int. J. Mod. Phys. D 18, 1025 (2009)

62. R. Pierce, J. Leitch, M. Stephens, P. Bender, R. Nerem, Appl. Opt. 47, 5007 (2008)

63. S.G. Turyshev, M.V. Sazhin, V.T. Toth, Phys Rev. D 89, 105029 (2014) 